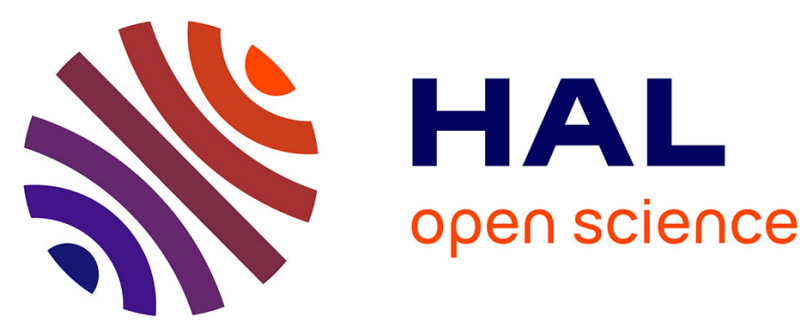

\title{
Thioflavine-T and Congo Red reveal the polymorphism of insulin amyloid fibrils when probed by polarization-resolved fluorescence microscopy.
}

Julien Duboisset, Patrick Ferrand, Wei He, Xiao Wang, Hervé Rigneault, Sophie Brasselet

\section{To cite this version:}

Julien Duboisset, Patrick Ferrand, Wei He, Xiao Wang, Hervé Rigneault, et al.. Thioflavine-T and Congo Red reveal the polymorphism of insulin amyloid fibrils when probed by polarization-resolved fluorescence microscopy.. Journal of Physical Chemistry B, 2013, 117 (3), pp.784-8. 10.1021/jp309528f . hal-00803617

\section{HAL Id: hal-00803617 https://hal.science/hal-00803617}

Submitted on 8 Oct 2014

HAL is a multi-disciplinary open access archive for the deposit and dissemination of scientific research documents, whether they are published or not. The documents may come from teaching and research institutions in France or abroad, or from public or private research centers.
L'archive ouverte pluridisciplinaire HAL, est destinée au dépôt et à la diffusion de documents scientifiques de niveau recherche, publiés ou non, émanant des établissements d'enseignement et de recherche français ou étrangers, des laboratoires publics ou privés. 


\title{
Thioflavine-T and Congo Red reveal the
}

\author{
polymorphism of insulin amyloid fibrils when
}

\section{probed by polarization-resolved fluorescence}

\section{microscopy}

Julien Duboisset, Patrick Ferrand, Wei He, Xiao Wang, Hervé Rigneault, Sophie Brasselet

Aix Marseille Université, CNRS, Ecole Centrale Marseille, Institut Fresnel, 13013 Marseille, France

Abstract: Amyloid fibrils are proteins misfolding structures that involve a $\beta$-sheet structure and are associated with the pathologies of various neurodegenerative diseases. Here we show that Thioflavine-T and Congo Red, two major dyes used to image fibrils by fluorescence assays, can provide deep structural information when probed by means of polarization resolved fluorescence microscopy. Unlike fluorescence anisotropy or fluorescence detected linear dichroism imaging, this technique allows to retrieve simultaneously both mean orientation and orientation dispersion of the dye, used here as a reporter of the fibril structure. We have observed that insulin amyloid fibrils exhibit a homogenous behavior over the fibrils' length, confirming their structural uniformity. In addition, these results reveal the existence of various structures among the observed fibrils' population, in spite of a similar aspect when imaged with conventional 
fluorescence microscopy. This optical non-destructive technique opens perspectives for in-vivo structural analyses or high throughput screening.

\section{Keywords}

Absorption dipole

protein aggregation

fluorophore distribution

confocal microscopy

rope-like structure

structural imaging 


\section{Introduction}

Protein aggregation and amyloid formation are widely studied due to their crucial role in a large number of pathologies such as Alzheimer's disease, Creustzfeldt-Jacob's disease or type II diabetes. However, the molecular scale organizations of amyloid that are responsible for toxicity $^{1,2}$ remain not completely understood due to the difficulty to crystallize such structures. Nevertheless, nuclear magnetic resonance ${ }^{3}$, x-ray diffraction ${ }^{4}$ and Raman spectroscopy ${ }^{5}$ allowed to determine the elementary amyloid organization as a $\beta$-sheet structure. In the case of amyloids based on the insulin polypeptide, its conformation changes at low $\mathrm{pH}$, which allows several molecules to assemble together in order to form soluble oligomers. During the fibrillation process, the oligomers have the ability to assemble themselves into protofilaments and filaments to reach fibrils organization, known as insulin amyloid ${ }^{6}$. Unfortunately, standard characterization techniques cannot address the additional complexity which comes from the wealth of molecular assemblies present at different scales.

Although it does not reach the power of structural analysis techniques, fluorescent microscopy is an established technique, especially for diagnostic purpose, because it allows to observe easily the formation of amyloid fibrils in tissues or in vitro ${ }^{7,8,9,10,11}$. In this context, much work has been carried out in order to synthesize efficient fluorescent probes that bind specifically to the amyloid structure $^{12,13,14}$. Historically, Congo Red $(\mathrm{CR})^{15}$ and later Thioflavine $\mathrm{T}(\mathrm{ThT})^{16}$ were the first fluorophores known for their specificity and are extensively used today. Confocal fluorescent anisotropy ${ }^{17}$ investigations, as well as atomic force microscopy (AFM) ${ }^{18}, \mathrm{x}$-ray ${ }^{19}$ and numerical simulations $^{20}$, allowed to determine the orientation of the fluorophores with respect to the fibril, and it came out that $\mathrm{CR}$ and ThT bind perpendicularly to the beta-sheet, i. e., parallel to the 
fibrils axis. Attempts to exploit the CR orientation in order to report the structural properties of amyloid have been reported using fluorescence detected linear dichroism imaging (FDLD) ${ }^{21}$. However, orientational information was only provided in sample areas where the average orientation could be a priori known unambiguously ${ }^{22-25}$. A more complete and flexible measurement scheme has been proposed recently, which circumvents these limitations. It is based on the comparison between fluorescence images recorded for more than two sequential excitation polarization angles and allows to quantify both the average orientation and the amplitude of angular the fluctuations of the fluorophores ${ }^{23,26,27}$. In this article, we propose to use a similar approach to probe the fluorescence of CR and ThT and provide a proof of concept of the ability to obtain structural information in amyloid fibrils.

\section{Methods}

The technique, that we name polarization-resolved fluorescence microscopy, but has been also refered to as excitation polarized fluorescence ${ }^{23}$, relies, like FDLD, on the angular selectivity of the absorption efficiency of a fluorescent molecule depending on its orientation, that is written $|\vec{\mu} \cdot \vec{E}|^{2}$, where $\vec{\mu}$ and $\vec{E}$ stand respectively for the absorbing dipole and the electric field of the excitation light ${ }^{24}$. The dot product states that absorption cannot occur when the field is perpendicular to the dipole, whereas it reaches a maximum when both vectors are parallel. If the angle $\alpha$ of the state of polarization of excitation light is sequentially rotated in the sample plane between 0 and 180 degrees (Fig. 1a), the analysis of the variations of fluorescence intensity versus a provides a non-ambiguous way to determine the orientation of the emitting dipoles that 
have been probed. As illustrated in Fig. 1, a sine response with a total cancellation of the fluorescence (Fig. 1b) is recorded when probing a dipole (or ensemble of dipoles) of given orientation (Fig. 1c), that is directly read at the maximum of intensity. A low contrast sine response (Fig. 1d) is however the signature of an ensemble of dipoles of various orientations averaged out over time and/or space. The angle $\alpha$ which gives the maximum intensity corresponds now to the average orientation $\rho$ of the dipoles in the sample plane. The loss of contrast of the response is related to the angular dispersion of the dipoles around this direction. We quantify this angular dispersion by an angle $\psi$. The distribution of molecules is thus simply modeled as a 3D filled cone, whose main symmetry axis lies in the XY plane, of full width $\psi$ (Fig. 1e) and orientation $\rho$. In order to resolve these two parameters unambiguously, polarization-resolved fluorescence imaging uses a modified confocal fluorescence microscopy system where a stack of fluorescence images is recorded under various excitation angles $\alpha$. Note that compare to FDLD, which uses a fast polarization modulation and lock-in detection at each pixel $^{21}$, our approach is significantly more time consuming but more generic since it provides a full control of various linear polarizations and allows to choose their angles $\alpha$ with a better flexibility. Using the analysis depicted above at each pixel of interest, both parameters $\rho$ and $\psi$ can be retrieved and mapped on the sample image. $\rho$ and $\psi$ are here retrieved independently, which constitutes a clear improvement compare to anisotropy fluorescence microscopy ${ }^{10,25}$ or FDLD $^{21}$, which rely in practice on the assumption on either $\rho$ or $\psi$. Note that since only the absorption efficiency is modulated, this technique, like FDLD, is intrinsically insensitive to depolarization effects such as those induced by energy transfer processes ${ }^{27}$. 
Technically, the retrieval is performed by comparing the recorded fluorescence signal $I(\alpha)$ to a set of theoretical responses computed ${ }^{26}$ for all possible sets $(\rho, \psi)$ using a similar formalism as what was developed for two-photon fluorescence ${ }^{27}$

$$
I(\alpha) \propto \iint|\vec{\mu}(\theta, \varphi) \cdot \vec{E}(\alpha)|^{2} f(\theta, \varphi) J(\theta, \varphi) \sin \theta d \theta d \varphi
$$

where the cone distribution is represented by $f(\theta, \varphi)$, i.e., a constant when $(\theta, \varphi)$ are inside the cone, zero else. The function $\mathrm{J}$ represents the detection probability of fluorescence and accounts for the collection by the objective lens ${ }^{24}$.

The experiments were carried out on a custom laser scanning fluorescence confocal microscope that was designed to provide a full control of the state of polarization of the excitation laser (continuous, 491-nm Calypso, Cobolt), that was reflected by a dichroic mirror (XF2037-500DRLP, Omega Optical) and focused onto the sample by a water immersion objective lens (C-Apochromat 40x, NA=1.2, Carl Zeiss), yielding to a typical lateral resolution of $200 \mathrm{~nm} .{ }^{28}$ Fluorescence was detected after passing through a band pass filter (HQ540/80M2P, Chroma). Data were recorded under a typical excitation power of $5 \mu \mathrm{W}$, with a pixel dwell time of $100 \mu \mathrm{s}$. The state of polarization for the excitation beam was controlled by rotating sequentially a half waveplate (WPH05M-488, Thorlabs) by means of a step motor in the path of the linearly polarized laser beam. Polarization distortions induced by the dichroic mirror were partially compensated using a wave plate and residual distortions were measured and taken into account in the theoretical computation used for data analysis ${ }^{26}$. Data were recorded by incrementing polarization angles $\alpha$ by steps of $2^{\circ}$ between 0 and $178^{\circ}$. Note that a much smaller number of steps (in principle down to 3 steps, i.e., $\alpha=0^{\circ}, 60^{\circ}$ and $120^{\circ}$ ) could be used. However, the reduction of data points must be compensated by larger dwell times and/or accumulations, in order to keep the necessary total photon counts that are required for a precise 
data analysis, namely a precision of a few degrees on both $\rho$ and $\psi$. Data processing includes selection of the region of interest by the user, systematic rejection of data points for which the photon counts is too low (typically below 300 photon counts), and systematic rejection of data points that were affected by sample drift or photobleaching ${ }^{26}$.

In the present study, the bovine insulin, ThT and CR were purchased from Sigma-Aldrich and used as received without further purification. Insulin was dissolved at a concentration of $2 \mathrm{mg} / \mathrm{mL}$ in acid buffer $(\mathrm{pH}=2,20 \%$ acetic acid, $100 \mathrm{mM} \mathrm{NaCl})$. Fibrillation was induced by heating the insulin solution at $50^{\circ} \mathrm{C}$ during $20 \mathrm{~h}$ without agitation. Then the solution was cooled down at room temperature and then centrifuged at $9500 \mathrm{G}$ for $10 \mathrm{~min}$ and redispersed in neat water several times to remove the excess of fluorescent probes. The ThT and CR were used at $0.25 \mu \mathrm{M}$, and inserted after the fibrillation process. The samples were analyzed immediately after their preparation, at room temperature.

\section{Results and discussion}

Typical polarization resolved fluorescent images from insulin amyloid fibrils labeled with CR and ThT are depicted in Fig. 2. A selection of fibrils with different orientations and fluorescent intensities is shown (using a red-yellow colorscale). For each sample, a composite image is displayed that summarizes for the relevant pixels the mean orientation $\rho$ (indicated by an oriented stick) and the cone aperture $\psi$ (indicated by the color of the stick) of the fluorophore as they have been retrieved by the method described above. The values of $\psi$ are summarized on a histogram. Note that structures of different diameters can be visible on the obtained images, 
representing a diversity of fibrils assemblies into more or less large structures, which we still denote as fibrils for simplicity. However for the polarization resolved data analysis, we focus here on the ones with a sufficiently high number of photons to ensure a high order determination precision, which corresponds to fibrils with a diameter close to $1 \mu \mathrm{m}$.

Remarkably, the average orientation $\rho$ of the fluorophores follows the overall orientation axis of the fibrils, in good agreement with previous results ${ }^{17,19,21,29}$. In Fig. 2a, this average orientation is uniform over the whole fibril, indicating that the supramolecular structure of the fibril, although it cannot be spatially resolved, remains the same over its total length, about $3 \mu \mathrm{m}$ here. On the contrary, in Fig $2 b$ (and close-up), although the overall orientation remains the same along the fiber, it appears clearly that local values of $\rho$ can vary along the fibril, showing that the method is sensitive to changes of orientation of only a few degrees.

Compared to $\rho$, which is an average value, $\psi$ carries determining information on the dispersion of the molecular orientations within the confocal spot of $200 \mathrm{~nm}$ size. On a given fibril, the values of $\psi$, similarly to what was observed for $\rho$, show a clear narrow distribution, suggesting the structural integrity of the fibril along its length, in agreement with previous observation performed using atomic force microscopy ${ }^{30}$. However, note that this macroscopic spatial homogeneity does not mean that the structure is structurally homogeneous at scales below the diffraction limit. Besides homogeneous regions, in some areas, an increase of $\psi$ suggests a localized structural defect, such as at the end of the fibril in Fig. 2b. More predictably, fibril crossings like in Fig. 2c mix two angular populations and show an artificially quasi isotropic signature. In all these cases, changes of $\psi$ were clearly correlated with a local variation of the mean orientation of the fluorophore. Note that since ThT (and CR, to a lesser extent) binds preferentially on the $\beta$-sheet structures, the technique is in principle only directly sensitive to the 
$\beta$-sheet distribution. Nevertheless, one could imagine that the presence of other structures may indirectly affect the morphology of the beta-sheet, and therefore the $\psi$ value.

Amongst the large variety of morphologies ${ }^{31}$, it is known that amyloid fibrils are formed in a relatively systematic fashion, in a rope-like structure, as illustrated in Fig. 3. Protofilaments assemble into pairs or larger groups by coiling around each other with a long-range twist arising from the small angle between their constituent beta-strands to form filaments. Therefore, the angles $\psi$ as they are measured by this method contain contributions at different spatial scales, starting from i) the intrinsic aperture $\psi_{0}$, that quantifies the angular freedom of the fluorophore that binds to the cross beta-sheet structure (Fig. 3a), ii) the angular aperture $\phi_{\mathrm{P}}$ between the strands of protofilaments forming the filament (Fig. 3b), up to iii) the angular distribution of the filaments $\phi_{\mathrm{F}}$ that build up a larger structure (Fig. 3c-e).

From cryo-electron microscopy measurements, it was shown that between two (Fig. 3b) and twelve protofilaments can assemble into filament ${ }^{32,33}$. Using the intercross section and the pitch angle of these assemblies, one can estimate that the projected angle of the protofilament into the filament structure $\phi_{\mathrm{P}}$ ranges between $20^{\circ}$ and $40^{\circ}$, depending on the number of protofilaments involved. This value can be exploited to deduce an upper limit for the intrinsic aperture $\psi_{0}$.

In the large set of fibril samples labeled with ThT or CR, the lowest measured value is $\psi \approx 80^{\circ}$ corresponding obviously to the most aligned filaments of the sample. This leads to a maximum value of $\psi_{0} \approx 60^{\circ}$. This value confirms the relatively narrow angle distribution that was predicted by theoretical works ${ }^{34}$. Since it quantifies the ability of the fluorophore to report on the cross beta sheet structure, it should be understood as the minimum structural angular aperture that can be measured using CR or ThT. The values of $\psi$ that were measured, ranging from 80 to 
$120^{\circ}$, are definitely much above this threshold, so they can effectively report on the fibril structure.

The sensitivity and the relevance of the parameter $\psi$ appear clearly when comparing Fig. $2 \mathrm{~b}$ to Fig. 2c. Fig. 2c shows two overlapping fibrils that are characterized by different values of $\psi$. The vertical fibril exhibits $\psi$ values around $82^{\circ} \pm 9^{\circ}$, while the oblique one shows values around $110^{\circ}$ $\pm 14^{\circ}$. These two fibrils, which have a similar aspect in fluorescence microscopy imaging, differ obviously by their structure, although it is not possible to claim through which of the angles $\phi_{\mathrm{P}}$ and/or $\phi_{\mathrm{F}}$. The oblique fibril appears actually very similar to the fibril of Fig. $2 \mathrm{~b}$, for which $\psi \approx 113^{\circ} \pm 10^{\circ}$. The observation of tens of fibrils in this sample showed the presence of these two populations of $\psi$ values. This shows that this technique could potentially allow a spatial discrimination of fibrils of different conformations.

As it was already mentioned, the average angle $\rho$ is aligned with the axis of the fibril. This is the signature of an assembly having an axial symmetry, such as rod bundles (Fig. 3c) or twisted fibrils (Fig. 3d). Very interestingly, the vertical fibril of Fig. 2c shows a $\rho$ angle that is tilted of about $15^{\circ}$ with respect to the fibril axis, an unambiguous signature of a parallel fibril assembly, such as the one illustrated in Fig. 3e.

\section{Conclusion}

We have demonstrated the potential of CR and ThT to be used as structural reporters of insulin amyloid fibrils, by implementing polarization resolved fluorescence microscopy. Although the method described here does not reach the power of structural analysis techniques, it provides 
new physical observables, namely the mean orientation $\rho$ and angular aperture $\psi$ of the fluorescent probe, that report on the structure of the fibrils. Moreover, it is compatible with in vivo measurements and dynamic observations, for instance to address transient intermediates structures. Thanks to its specificity and sensitivity, it could open new perspectives for high throughput screening, aiming at a better understanding of amyloids and related diseases. More generally, it can address the issue of how fluorophores are oriented in supramolecular structures.

\section{Corresponding Author}

* J. D. (Email: julien.duboisset@fresnel.fr)

\section{ACKNOWLEDGMENT}

This research was supported by the Région Provence Alpes Côte d'Azur and by the French Agence Nationale de la Recherche under contract ANR-2010-BLAN-150902. H. W. received an Erasmus Mundus scholarship under contract 2010-2462/001-001/EMMC/Europhotonics. X. W. received a scholarship from the China Scholarship Council. 


\section{FIGURE CAPTIONS}

Figure 1: (a) Scheme of the polarization resolved fluorescence microscopy setup. The close-up shows the sample plane where the state of polarization of the excitation light is rotated sequentially with an angle $\alpha$ during the data acquisition. (b) Two cases (theoretical plots) of fluorescence signal versus $\alpha$, and (c) corresponding dipole orientations. (d) Theoretical plots for an angular distribution of dipoles in the plane, and (e) retrieved distributions.

Figure 2: Polarization-resolved fluorescence measurements performed on fibrils labeled with CR (a) and $\operatorname{ThT}(b, c)$. For each case, the red/yellow colormap indicates the total fluorescence intensity recorded. The composite image summarizes the mean orientation $\rho$ (indicated by an orientated stick) and the angular aperture $\psi$ (ranging from 0 to $180^{\circ}$, encoded as a color) of the fluorophores as they have been measured by the method. A histogram gives an overview of the distribution of the obtained $\psi$ values. All scale bars are $1 \mu \mathrm{m}$.

Figure 3: Schematic structure of amyloid fibrils, at different scales and their corresponding angular distributions. a) Beta-sheet structure showing the intrinsic aperture of the fluorophore. The grey lines represent insulin polypeptides. b) Protofilaments structure of two beta-sheet ribbons. c) Rod bundles fibrils distribution. d) Twisted fibrils distribution. e) Parallel fibrils distribution. 

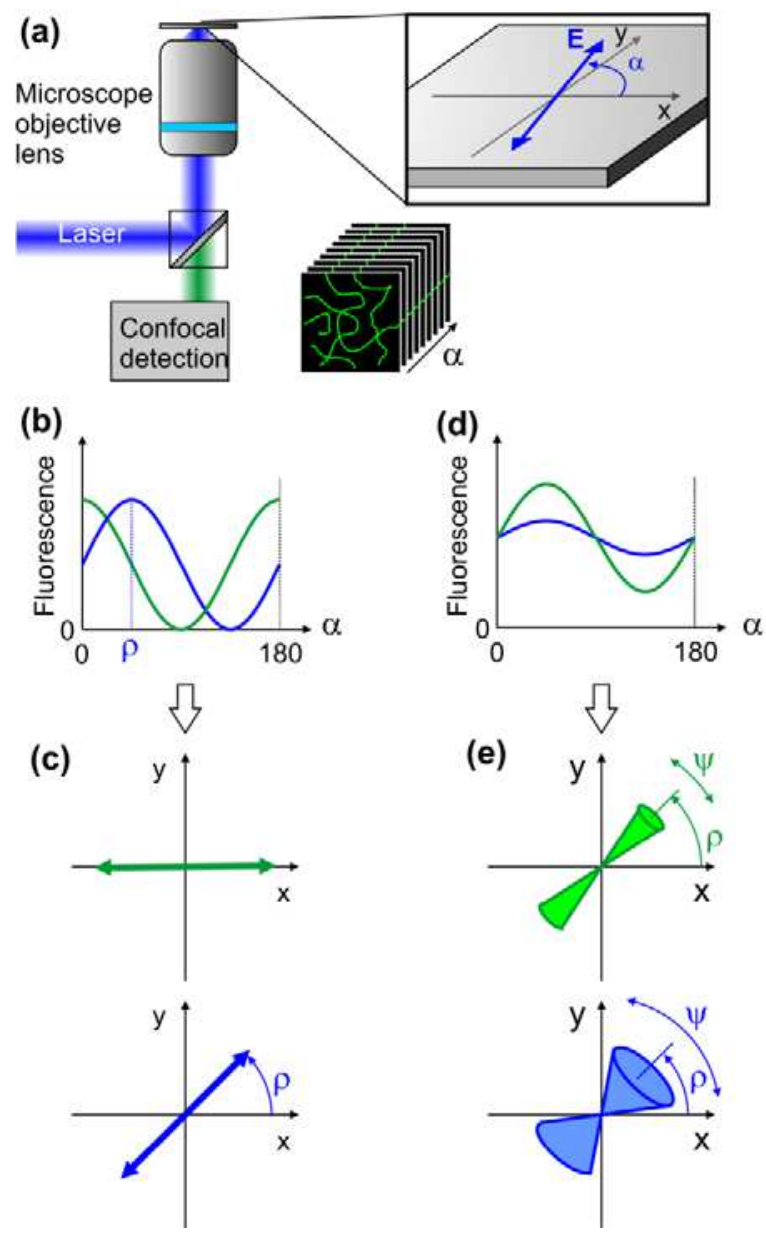

Figure 1 

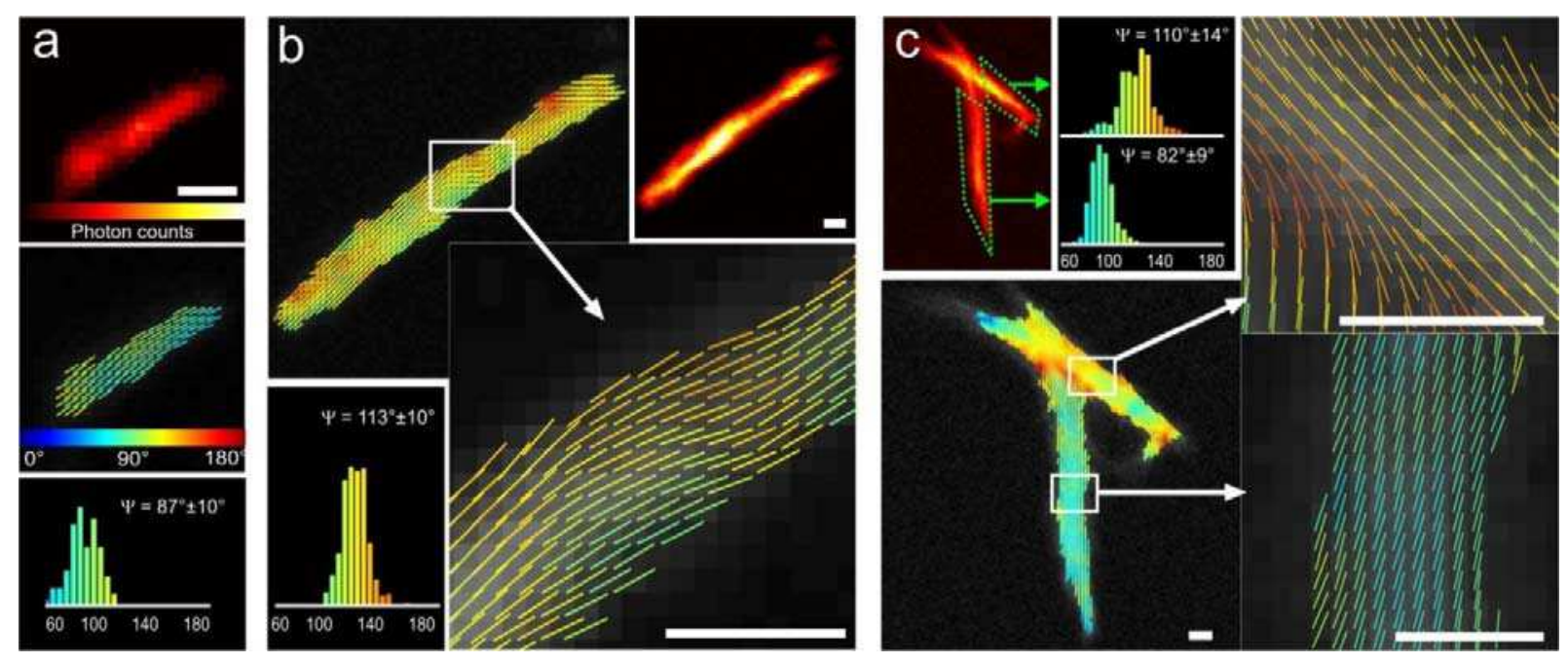

Figure 2 


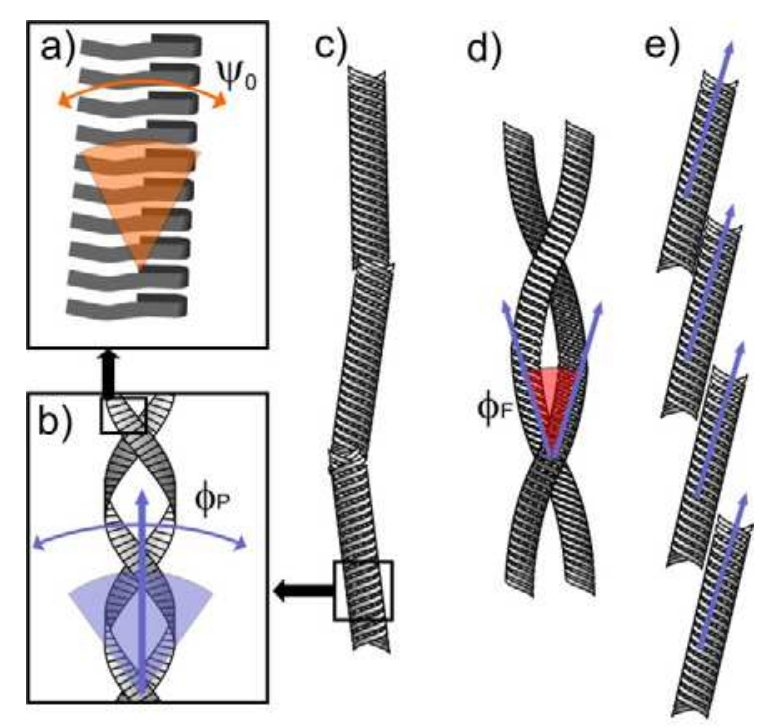

Figure 3 
TOC Figure

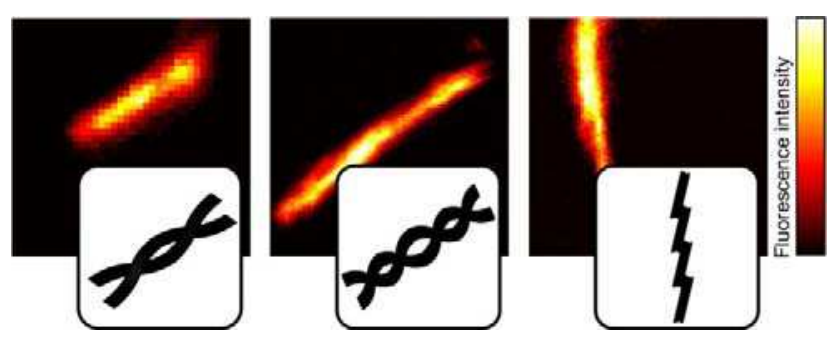




\section{REFERENCES}

${ }^{1}$ Lorenzo, A.; Yankner, B. A. Proc. Natl. Acad. Sci. U. S. A. 1994, 91 (25), 12243-12247.

${ }^{2}$ Seilheimer, B.; Bohrmann, B.; Bondolfi, L.; Muller, F.; Stuber, D.; Dobeli, H. J. Struct. Biol. 1997, 119 (1), 59-71.

${ }^{3}$ Petkova, A. T.; Ishii, Y.; Balbach, J. J.; Antzutkin, O. N.; Leapman, R. D.; Delaglio, F.; Tycko, R. Proc. Natl. Acad. Sci. U. S. A. 2002, 99 (26), 16742-16747.

${ }^{4}$ Blake, C.; Serpell, L. Structure 1996, 4 (8), 989-998.

${ }^{5}$ Popova, L. A.; Kodali, R.; Wetzel, R.; Lednev, I. K. J. Am. Chem. Soc. 2010, 132 (18), 63246328.

${ }^{6}$ Nettleton, E. J.; Tito, P.; Sunde, M., Bouchard, M.; Dobson, C. M.; Robinson, C. V. Biophys. J. 2000, 79 (2), 1053-1065.

${ }^{7}$ Kaminski Schierle, G. S.; van de Linde, S.; Erdelyi, M.; Esbjorner, E. K.; Klein, T.; Rees, E.; Bertoncini, C. W.; Dobson, C. M.; Sauer, M.; Kaminski, C. F. J. Am. Chem. Soc. 2011, 133 (33), 12902-12905.

${ }^{8}$ Kitts, C. C.; Bout, D. A. V. J. Phys. Chem. B 2009, 113 (35), 12090-12095.

${ }^{9}$ Psonka-Antonczyk, K. M. et al. Int. J. Mol. Sci. 2012, 13 (2), 1461-1480.

${ }^{10}$ Roberti, M. J.; Jovin, T. M.; Jares-Erijman, E. PLoS One 2011, 6, e23338.

${ }^{11}$ Bouchard, M.; Zurdo, J.; Nettleton, E. J.; Dobson, C. M.; Robinson, C. V. Protein Sci. 2000, 9 (10), 1960-1967.

${ }^{12}$ Lindgren, M.; Hammarstrom, P. FEBS Journal 2010, 277 (6), 1380-1388.

${ }^{13}$ LeVine, H. Biochemistry 2005, 44 (48), 15937-15943.

${ }^{14}$ Bertoncini, C. W.; Celej, M. S. Curr. Protein Pept. Sci. 2011, 12 (3), 206-220. 
${ }^{15}$ Khurana, R.; Uversky, V. N.; Nielsen, L.; Fink, A. L. J. Biol. Chem. 2001, 276 (25), 2271522721.

${ }^{16}$ Levine, H. Protein Sci. 1993, 2 (3), 404-410.

${ }^{17}$ Krebs, M. R. H.; Bromley, E. H. C.; Donald, A. M. J. Struct. Biol. 2005, 149 (1), 30-37.

${ }^{18}$ Khurana, R.; Coleman, C.; Ionescu-Zanetti, C.; Carter, S. A.; Krishna, V.; Grover, R. K.; Roy, R.; Singh, S. J. Struct. Biol. 2005, 151 (3), 229-238.

19 Groenning, M.; Norrman, M.; Flink, J. M.; van de Weert, M.; Bukrinsky, J. T.; Schluckebier, G.; Frokjaer, S. J. Struct. Biol. 2007, 159 (3), 483-497.

${ }^{20}$ Carter, D. B.; Chou, K. C. Neurobiol. Aging 1998, 19 (1), 37-40.

${ }^{21}$ Steinbach, G.; Pomozi, I.; Zsiros, O.; Menczel, L.; Garab, G. Acta Histochem 2009, 111, 316-325.

${ }^{22}$ Steinbach, G.; Pomozi, I.; Janosa, D. P.; Makovitzky, J.; Garab, G. J. Fluoresc. 2011, 21(3), 983-989.

${ }^{23}$ DeMay, B.S.; Noda, N.; Gladfelter, A.S.; Oldenburg, R. Biophys. J. 2011, 101, 985-994.

${ }^{24}$ Axelrod, D. Biophys. J. 1979, 26, 557-573.

${ }^{25}$ Rocheleau, J. V.; Edidin, M.; Piston, D. W. Biophys. J. 2003, 84, 4078-4086.

${ }^{26}$ Kress, A.; Wang, X.; Ranchon, H.; Savatier, J.; Rigneault, H.; Ferrand, P.; Brasselet, S. submitted (2012).

${ }^{27}$ Gasecka, A.; Han, T. J.; Favard, C.; Cho, B. R.; Brasselet, S. Biophys. J. 2009, 97, 28542862.

${ }^{28}$ Ferrand, P.; Pianta, M.; Kress, A.; Aillaud, A. Rigneault, H.; Marguet, D. Rev. Sci. Instrum. 2009, 80, 083702. 
${ }^{29}$ Kaminsky, W.; Jin, L. W.; Powell, S.; Maezawa, I.; Claborn, K.; Branham, C.; Kahr, B. Micron 2006, 37, 324.

${ }^{30}$ Knowles, T. P. J.; Smith, J. F.; Craig, A.; Dobson, C. M.; Welland, M. E. Phys. Rev. Lett. 2006, 96, 238301.

${ }^{31}$ Jansen, R.; Dzwolak, W.; Winter, R. Biophys. J. 2005, 88 (2), 1344-1353.

${ }^{32}$ Jimenez, J. L.; Nettleton, E. J.; Bouchard, M.; Robinson, C. V.; Dobson, C. M.; Saibil, H. R. Proc. Natl. Acad. Sci. U. S. A. 2002, 99 (14), 9196-9201.

${ }^{33}$ Meinhardt, J.; Sachse, C.; Hortschansky, P.; Grigorieff, N.; Faendrich, M., J. Mol. Biol. 2009, $386(3), 869-877$.

${ }^{34}$ Biancalana, M.; Koide, S. Biochim. Biophys. Acta, Proteins Proteomics 2010, 1804 (7), $1405-1412$. 